\title{
Força Muscular Versus Pressão Arterial \\ de Repouso: Uma Revisão Baseada no Treinamento Com Pesos
}

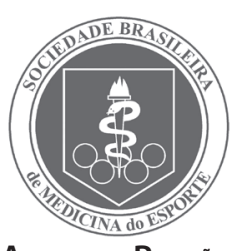

Artigo de Revisão

\section{Muscular Strength versus Blood Pressure at Rest: A Review Based on Weight Training}

Marcos Doederlein Polito

Universidade Estadual de Londrina

- Centro de Educação Física

e Esporte - Departamento de

Educação Física.

Endereço para correspondência: Rodovia Celso Garcia Cid, km 380

- Campus Universitário

86051-901 - Londrina, PR:.

Tel.: (43) 3371-4238.

E-mail: marcospolito@uel.br

Submetido em 28/08/2008

Versão final recebida em 02/11/2008

Aceito em 08/12/2008

\section{RESUMO}

Introdução: O exercício com pesos é atualmente recomendado para diferentes grupos de indivíduos. Seu principal objetivo é o aumento na força muscular, mas podem ocorrer outros resultados, como a redução na pressão arterial (PA) de repouso. Contudo, é pouco abordada a relação entre prescrição do treinamento com pesos, aumento da força e comportamento da PA de repouso em humanos. Objetivo: Analisar a PA após uma sessão aguda de exercícios com pesos e após um período de treinamento com pesos, em indivíduos normotensos e hipertensos, em estudos publicados na literatura internacional. Métodos: As referências foram selecionadas de três formas distintas: 1) para compor as sugestões de treinamento de pesos foi utilizado um estudo meta-analítico e um posicionamento oficial de uma reconhecida entidade de pesquisa; 2) para os dados do exercício com pesos e PA de repouso nos momentos pós-exercício, foram pesquisados estudos na base Medline publicados em inglês que acompanharam a PA por pelo menos 60 min; 3) as referências sobre treinamento com pesos em longo prazo e PA de repouso foram resgatadas do último estudo meta-analítico sobre o tema, adicionadas dos experimentos publicados até julho de 2008. Resultados: Após analisar as referências, a prescrição do treinamento com pesos, na maioria dos estudos, seguiu sugestões atuais. Porém, somente em poucos estudos a PA de repouso foi reduzida, tanto em normotensos quanto em hipertensos. Conclusões: Mesmo com certa variação na prescrição, o treinamento com pesos parece ser suficiente para aumentar a força muscular. Contudo, ainda faltam pesquisas para inferir sobre sua relação com a PA de repouso de normotensos e hipertensos.

Palavras-chave: resposta cardiovascular, hipertensão, treinamento de força.

\begin{abstract}
Introduction: Weight training is currently recommended to different groups of individuals. Its main objective is increase in muscular strength, but other results may occur, such as blood pressure (BP) at rest reduction. However, the relationship among weight training prescription, increase in strength and BP behavior at rest in humans is little approached. Aim: To analyze BP after an acute session of weight exercises and after a weight training period in normotensive and hypertensive individuals, in studies published in the international literature. Methods: References were selected in three distinct ways: 1) in order to compose the weight training suggestions, a meta-analytical and an official positioning of a recognized research entity was used; 2) for the weight exercise and BP at rest data at the post-exercise moments, studies from the Medline basis published in English which followed BP for at least 60 min were researched; 3 ) references on long term-weight training and BP at rest were retrieved from the last metaanalytical study on the topic, added to the experiments published until July, 2008. Results: After analyzing the references, prescription of weight training in most of the studies followed current recommendations. Nevertheless, only in few studies BP at rest was reduced, both in normotensive and hypertensive subjects. Conclusions: Despite certain variation in prescription, weight training seems to be efficient in increasing muscular strength. However, further research is still needed in order to infer on its correlation with BP at rest in normotensive and hypertensive subjects.
\end{abstract}

Keywords: cardiovascular response, hypertension, strength training. 


\section{INTRODUÇÃO}

Atualmente, entidades de pesquisas internacionais e nacionais relacionadas com a saúde indicam o treinamento com pesos como parte integrante de um programa de exercícios físicos para auxiliar no controle da pressão arterial (PA) de repouso ${ }^{(1,2)}$. Tal controle pode ocorrer de forma crônica, proveniente do treinamento sistemático, ou de forma imediata, proveniente de uma única sessão de exercícios, através do efeito denominado hipotensão pós-exercício ${ }^{(3)}$. Mais ainda, os benefícios do exercício com pesos sobre os valores de repouso da PA podem ser observados tanto em sujeitos hipertensos (de forma imediata ${ }^{(4)}$ ou crônica $\left.{ }^{(5)}\right)$, quanto normotensos (igualmente de forma imediata ${ }^{(6)}$ ou crônica $^{(7)}$ ).

Porém, independentemente dos resultados do treinamento com pesos sobre o sistema cardiovascular, o objetivo principal dessa modalidade física é aumentar a força muscular. Em um indivíduo hipertenso, por exemplo, o aumento da força muscular pode representar menor estresse cardiovascular em um esforço físico ${ }^{(8)}$. Porém, o aumento da força muscular depende de estratégias de prescrição relacionadas com variáveis como carga, número de repetições, número de séries e intervalo de descanso ${ }^{(9)}$.

Devido à grande variação de possibilidades para prescrição dos exercícios com pesos, os estudos que investiram nesse treinamento com finalidade de redução da PA de repouso adotaram metodologias diferenciadas, impossibilitando uma comparação direta entre o comportamento da PA e o aumento da força. Por exemplo, a hipotensão pós-exercício (HPE) pode se manifestar após o exercício com pesos realizado em alta ou baixa intensidade em pessoas normotensas ${ }^{(10)}$ e hipertensas ${ }^{(4)}$. A continuidade do treinamento, entretanto, poderia repercutir de forma diferenciada sobre a força muscular, ocasionando ganhos diferenciados de acordo com a intensidade adotada. Sobre o treinamento em longo prazo, dados meta-analíticos indicam a possibilidade de redução da PA de repouso após períodos de treinamento com pesos em hipertensos e normotensos, igualmente sob variados delineamentos de prescrição, mas não fazem alusão ao comportamento da força muscular ${ }^{(11,12)}$.

Dessa forma, torna-se importante uma abordagem que relacione o comportamento da PA de repouso com as variáveis do treinamento com pesos que poderiam estimular o aumento da força muscular. Assim, o objetivo do presente estudo foi analisar o comportamento da PA após uma sessão aguda de exercícios com pesos e após um período de treinamento físico com pesos, em indivíduos normotensos e pacientes hipertensos em estudos publicados na literatura internacional no período de 1987 a 2008.

\section{Métodos de busca e inclusão de artigos}

A inclusão de artigos foi determinada para contemplar três aspectos do presente estudo: a) prescrição do treinamento com pesos para aumento da força muscular; b) treinamento com pesos relacionado com a HPE em humanos; c) treinamento com pesos em longo prazo e PA de repouso em humanos. No primeiro caso, foram utilizadas referências de metaanálise ${ }^{(13)}$ e do posicionamento do Colégio Americano de Medicina do Esporte ${ }^{(9)}$, por se tratar de abordagens com pouca variação metodológica de prescrição. No segundo caso, foram pesquisados estudos na base Medline através das palavras-chave post-exercise hypotension, resistance exercise, strength exercise, resistive exercise, weight training, blood pressure response e recovery blood pressure no título ou no resumo. Foram considerados válidos os estudos que acompanharam a PA pós-exercício pelo menos por 60 min. No terceiro caso, foram utilizados os artigos incluídos na última meta-análise publicada sobre exercício com pesos e PA de repouso ${ }^{(12)}$. No entanto, como a referida meta-análise utilizou experimentos até 2003, foram pesquisados estudos publicados de 2003 até julho de 2008 na base Medline com as palavras-chave blood pressure, resting blood pressure, cardiovascular response, resistance training, strength training, resistive training, resistance exercise, strength exercise, resistive exercise, normotensive subjects, hypertensive subjects e hypertension. Considerando a quantidade relativamente reduzida de experimentos sobre exercício com pesos e PA de repouso, novas referências foram incluídas sem o delineamento aleatorizado, mas envolvendo grupo controle e duração mínima de quatro semanas.

\section{Prescrição do treinamento com pesos para aumento da força muscular}

O treinamento com pesos possui diversas variáveis associadas à prescrição, como carga, número de repetições, número de séries, intervalo de repouso, frequência semanal, velocidade de execução, quantidade de exercícios, tipo de exercício (uniarticular ou multiarticular), ordem dos exercícios e tipo de ação muscular. O controle de todas as variáveis pode ser necessário quando o objetivo do treino é maximizar o desempenho ${ }^{(14)}$. Em se tratando do aumento da força muscular para questões de saúde e qualidade de vida, a atenção pode ser destinada a apenas algumas dessas variáveis. Porém, é importante considerar que a força muscular destinada à saúde deve ser suficiente para executar tarefas cotidianas e de lazer com eficiência ${ }^{(15)}$. Portanto, o treinamento com pesos para essa finalidade deve atender a pressupostos metodológicos descritos na literatura.

O Colégio Americano de Medicina do Esporte publicou dois posicionamentos sugerindo a prescrição do treinamento com pesos ${ }^{(9,16)}$. Para o presente estudo, foi considerado o documento mais recente ${ }^{(9)}$, por ser exclusivo do treinamento com pesos e descriminar mais variáveis. Nesse posicionamento, por exemplo, a amostra é dividida de acordo com o estado de treinamento: a) iniciante: sem experiência no treinamento com pesos; b) intermediário: treinamento regular pelo menos por seis meses; c) avançado: treinamento regular por mais de um ano. Obviamente, os ajustes fisiológicos e neuromotores que mediam a força muscular podem variar individualmente.

As demais variáveis do posicionamento do Colégio Americano de Medicina do Esporte ${ }^{(9)}$ estão descritas na tabela 1. A sugestão original abrange outras manifestações da força (como potência muscular, por exemplo). Contudo, em termos relacionados com a saúde, a literatura sugere o aumento da força per se e da resistência muscular. Sutis, porém importantes diferenças entre os objetivos podem ser observadas na carga, no número de repetições e no intervalo de recuperação. Dessa forma, o resultado de um treinamento visando exclusivamente a força muscular deve proporcionar ajustes neuromotores para recrutar fibras de contração rápida e modificações intramusculares de aumento de conteúdo contrátil. Por outro lado, o treinamento de resistência muscular relaciona-se com a tolerância à fadiga.

Tabela 1. Recomendações do Colégio Americano de Medicina do Esporte ${ }^{(9)}$ para prescrição do treinamento com pesos

\begin{tabular}{|c|c|c|c|c|c|c|}
\hline Amostra & Objetivo & Número de séries & Número de repetições & Carga (\%1RM) & Frequência semanal & Tempo de recuperação \\
\hline Iniciantes & Força & $1-3$ & $8-12$ & $60-70 \%$ & $2-3$ & $1-3 \mathrm{~min}$ \\
\hline Intermediários & & $>3$ & $6-12$ & $70-80 \%$ & $2-4$ & $1-3 \min$ \\
\hline Treinados & & $>3$ & Até 12 & $70-100 \%$ & $4-6$ & $1-3 \min$ \\
\hline Iniciantes & Resistência & $1-3$ & $10-15$ & $50-70 \%$ & $2-3$ & 1-2 min ou menos \\
\hline Intermediários & & $>3$ & $10-15$ & $50-70 \%$ & $2-4$ & 1-2 min ou menos \\
\hline Treinados & & $>3$ & $10-25$ ou mais & $30-80 \%$ & $4-6$ & $1-2$ min ou menos \\
\hline
\end{tabular}


Além do posicionamento do Colégio Americano de Medicina do Esporte ${ }^{(9)}$, o estudo de Rhea et al.(13) foi considerado como referência por ser uma importante meta-análise sobre treinamento com pesos e força muscular, e não apenas uma sugestão de treino. Nesse trabalho, os autores descrevem a amostra apenas de duas formas: destreinados (menos de um ano de treinamento regular) e treinados (mais de um ano de treinamento regular). As variáveis analisadas pelos autores são descritas na tabela 2. Nesse estudo ${ }^{(13)}$, os autores consideraram o aumento da força isoladamente, sem comparação entre suas manifestações. A principal diferença entre os grupos analisados relaciona-se à carga e às repetições. Isso se deve, principalmente, ao fato de os sujeitos menos treinados responderem com maior magnitude, mesmo com cargas relativamente menores ${ }^{(17)}$. Tais dados concordam com os do Colégio Americano de Medicina do Esporte ${ }^{(9)}$. O número de séries das pessoas iniciantes pode ser o maior desacordo entre as referências. O Colégio Americano de Medicina do Esporte ${ }^{(9)}$ sugere entre uma e três séries, enquanto Rhea et al. ${ }^{(13)}$ propõem quatro. Isso poder ser explicado pela diferença entre o que foi considerado como iniciante pelo Colégio Americano de Medicina do Esporte ${ }^{(9)}$ - menos de seis meses de treino - e por Rhea et al. ${ }^{(13)}$ - menos de 12 meses de treino. Além disso, Rhea et al. ${ }^{(13)}$ consideram as séries por grupo muscular e não por exercício, como o Colégio Americano de Medicina do Esporte ${ }^{(9)}$.

Tabela 2. Sugestão para a prescrição do treinamento com pesos com intuito de aumentar a força muscular de sujeitos sedentários e treinados de acordo com Rhea et al.(113)

\begin{tabular}{l|c|c|c|c}
\hline \multicolumn{1}{c|}{ Amostra } & $\begin{array}{c}\text { Número de séries por } \\
\text { grupo muscular }\end{array}$ & $\begin{array}{c}\text { Número de } \\
\text { repetições }\end{array}$ & $\begin{array}{c}\text { Carga } \\
\text { (\%1RM) }\end{array}$ & $\begin{array}{c}\text { Frequência } \\
\text { semanal }\end{array}$ \\
\hline Sedentários & 4 & Até 12 & $60 \%$ & 3 \\
\hline Treinados & 4 & Até 8 & $80 \%$ & 2 \\
\hline
\end{tabular}

\section{Exercício com pesos e hipotensão pós-exercício}

Foram encontradas 14 referências sobre exercício com pesos e HPE (tabela 3). Dessas, 11 utilizaram sujeitos normotensos como amostra ${ }^{(10,18-27)}$; duas, sujeitos hipertensos ${ }^{(4,28)}$; e uma, hipertensos e normotensos ${ }^{(6)}$. Assim, foram totalizados 12 estudos com normotensos e três com hipertensos.

Dos 12 estudos com normotensos, a PA sistólica (PAS) reduziu-se significativamente após o exercício em quatro estudos $(6,10,24,26)$ e aumentou significativamente em um estudo(23). A queda na PAS variou entre 40 e $90 \mathrm{~min}$, enquanto o aumento perdurou por $15 \mathrm{~min}$. O aumento na PAS pós-exercício não seria esperado em termos fisiológicos. No entanto, os autores $^{(23)}$ também investigaram questões relacionadas com o estado de ansiedade da amostra, fato que pode ter influenciado o comportamento cardiovascular. Em relação à prescrição do treinamento, os estudos que observaram redução pós-exercício na PAS utilizaram diferentes metodologias, compreendendo séries intensas e moderadas, inclusive com execução em circuito. De forma geral, tais prescrições concordam com as sugestões referenciadas para o aumento da força muscular ${ }^{(9,13)}$. Há de se considerar, também, que não houve diferença relevante entre os estudos que reportaram dois modelos de prescrição diferenciados. Por exemplo, Rezk et al.(24) observaram redução na PAS por 90 min após o treinamento realizado com intensidade alta e baixa. De forma semelhante, Simão et al. ${ }^{(10)}$ encontraram reduções na PAS em diferentes intensidades por períodos muito próximos de duração. Somente no estudo de MacDonald et al. ${ }^{(26)}$ a prescrição do exercício não seguiu modelos convencionais. Nesse caso, a amostra realizou um único exercício para membros inferiores sem interrupção, exercitando somente uma perna. Quando esta se fatigava, o sujeito trocava imediatamente a perna, e assim sucessivamente durante 15 min. Essa estratégia, embora eficiente para ocasionar HPE, perde validade externa, pois é pouco aplicável em termos de aumento de força muscular. Por isso, dos quatro estudos que identificaram redução na PAS após o exercício, somente três ${ }^{(6,10,24)}$ (25\% do total de experimentos) utilizaram modelo de prescrição recomendado para o aumento da força muscular. Dessa forma, considerando os achados relacionados com a redução da PAS pós-exercício em normotensos, parece que sua observação não é frequente. No entanto, quando se manifesta, a queda parece independer da intensidade do esforço.

Em relação à PA diastólica (PAD), houve redução significativa também em quatro estudos ${ }^{(10,22,24,25)}$. No estudo de Bermudes et al. ${ }^{(19)}$, a PAD reduziu-se apenas durante o sono. Somente no estudo de Hill et al. ${ }^{(25)}$ o comportamento da PAD perdurou por 60 min. Nos demais ${ }^{(10,22,24)}$, a redução ocorreu por muito pouco tempo, variando de 30 min $^{(22)}$ e menos de 15 min $^{(10,24)}$. Nos estudos de Rezk et al. ${ }^{(24)}$ e Simão et al. ${ }^{(10)}$, inclusive, a queda da PAD ocorreu quando a amostra realizou exercícios de baixa intensidade, enquanto nos demais as repetições foram realizadas até a exaustão com intensidade próxima de 70\% de uma repetição máxima (RM). Assim, de forma semelhante ao reportado para a PAS, a queda pós-exercício na PAD em normotensos não deve ser frequente. Quando existe, porém, o tempo de duração parece ser curto e os dados são insuficientes para relacionar sua redução com alguma variável da sessão de exercícios.

Em se tratando da amostra hipertensa, três estudos ${ }^{(4,6,28)}$ compararam a PA após o exercício com pesos. Em todos foram observadas reduções significativas na PAS entre 60 e 0 min. Contudo, um estudo observou redução na PAD por pouco tempo no ambiente laboratorial, mas identificou redução na PAS e PAD durante o período de vigília por monitorização ambulatorial(4). Em relação à prescrição do exercício com pesos para hipertensos, foi verificada variação entre os estudos. No entanto, por se tratar de pessoas hipertensas, dois estudos ${ }^{(4,6)}$ optaram por cargas mais moderadas, no limite inferior da recomendação do Colégio Americano de Medicina do Esporte ${ }^{(9)}$. O exercício de baixa intensidade é importante para evitar aumentos exagerados da PA durante sua realização. Devido à escassez de pesquisas com pessoas hipertensas, maiores inferências sobre o comportamento da PA pós-exercício em hipertensos seriam apenas especulativas. Porém, os resultados até o momento podem ser tidos como promissores.

Para além desses comentários, é importante considerar que a implicação clínica da HPE relaciona-se com o maior tempo possível de redução da PA. Dessa forma, a monitorização deveria ser realizada de forma ambulatorial, liberando a amostra para as suas atividades diárias. Contudo, a maioria dos experimentos mediu a PA pós-exercício em ambiente laboratorial, com a amostra imóvel e controlada. Mais ainda, são poucos os estudos com sujeitos hipertensos e os resultados dos estudos com normotensos não podem ser generalizados. Assim, é necessário maior investimento nesse campo de pesquisa.

Finalmente, a maioria dos estudos que observaram HPE tanto em normotensos quanto em hipertensos seguiram as recomendações de prescrição do exercício com pesos ${ }^{(9,13)}$. Assim, em longo prazo, além do possível benefício cardiovascular, pode-se esperar aumento significativo de força relacionado com a saúde.

\section{Treinamento com pesos e pressão arterial de repouso}

Foram encontradas 12 referências sobre o treinamento com pesos em longo prazo e a PA de repouso em normotensos ${ }^{(7,29-39)}$ e seis referências em hipertensos ${ }^{(5,32,40-43)}$. Dos estudos com normotensos, duas referências possuíam dois grupos experimentais ${ }^{(35,37)}$. Dessa forma, envolvendo normotensos, foram considerados 14 grupos de treinamento. Desses grupos envolvendo normotensos, 11 reportaram aumento significativo da força muscular (7,29,30,32,34-37,39); cinco, redução na PAS(7,31,35,37); quatro, redução na $P A D^{(7,31,35)}$. Os quatro que reportaram redução na $P A D$ também relataram redução na PAS. Somente três grupos reportaram simultaneamente redução na PAS e PAD e aumento na força muscu$\operatorname{lar}^{(7,35)}$. É importante considerar que um estudo reportou redução na 
Tabela 3. Comportamento da pressão arterial até 24h após o exercício com pesos

\begin{tabular}{|c|c|c|c|c|c|c|c|c|}
\hline Referência & Amostra & N & $\begin{array}{l}\text { Monitorização } \\
\text { pós exercício }\end{array}$ & $\begin{array}{l}\text { Número de } \\
\text { exercícios }\end{array}$ & Séries & Repetições & Intensidade & Resultados \\
\hline & Normotensos & & & & & & & \\
\hline Roltsch et al. ${ }^{18}$ & $\begin{array}{c}\text { Treinados }(23 \pm 2 \text { anos }), \\
\text { sedentários }(20 \pm 2 \text { anos }) \\
\text { e treinados aerobiamente } \\
(24 \pm 3 \text { anos })\end{array}$ & $M=15 ; F=18$ & $24 \mathrm{~h}$ & 12 & 2 & $8-12$ & Máxima (8-12RM) & NS \\
\hline Bermudes et al. ${ }^{19}$ & Sedentários (44 \pm 1 anos) & $M=25$ & $24 \mathrm{~h}$ & 10 (circuito) & 3 & $20-35$ & $40 \% 1 \mathrm{RM}$ & Redução da PAD durante o sono \\
\hline Niemelä et a..20 & Ativos (31 \pm anos) & $M=12$ & $180 \mathrm{~min}$ & 4 & 3 & 12 e 20 & $\begin{array}{l}80 \% 1 \mathrm{RM} \text { (12 rep) e } \\
30 \% 1 \mathrm{RM} \text { (20 rep) }\end{array}$ & $\mathrm{NS}^{*}$ \\
\hline Focht e Koltyn ${ }^{21}$ & Treinados e sedentários & 84 & $180 \mathrm{~min}$ & 4 & 3 & $4-8$ e $12-20$ & $\begin{array}{l}80 \% 1 \text { RM (4-8 rep) e } \\
50 \% 1 \text { RM (12-20 rep) }\end{array}$ & NS \\
\hline DeVan et al..$^{22}$ & $\begin{array}{l}\text { Ativos e sedentários } \\
\quad(27 \pm 1 \text { anos })\end{array}$ & $M=11 ; F=5$ & $150 \mathrm{~min}$ & 9 & 1 & Máximas & $75 \% 1 \mathrm{RM}$ & Redução na PAD 30 min pós-exercício \\
\hline O'Connor et al. ${ }^{23}$ & Ativos ( $23 \pm 4$ anos) & $F=14$ & $120 \mathrm{~min}$ & 6 & 3 & 10 & 40,60 e $80 \% 10 R M$ & $\begin{array}{l}\text { PAS mais elevada após } 15 \text { min da } \\
\text { intensidade de } 80 \%\end{array}$ \\
\hline Rezk et al. ${ }^{24}$ & Sedentários (23 \pm 1 anos) & $M=8 ; F=9$ & $90 \mathrm{~min}$ & 6 & 3 & 10 e 20 & $80 \%$ 1RM e $40 \% 1 \mathrm{RM}$ & $\begin{array}{c}\text { Redução na PAS em ambas as intensidades } \\
\text { por } 90 \text { min; redução na PAD entre } 15^{\circ} \text { e } \\
30^{\circ} \text { minuto na intensidade } 40 \% \\
\end{array}$ \\
\hline Hill et al. ${ }^{25}$ & Treinados (22-33 anos) & $M=6$ & $60 \mathrm{~min}$ & 4 (circuito) & 3 & Máximas & $70 \% 1 \mathrm{RM}$ & Redução para a PAD nos 60 min. \\
\hline Simão et al..$^{10}$ & $\begin{array}{l}\text { Dois grupos treinados } \\
\qquad(\mathrm{G} 1=22 \pm 4 \text { anos; } \\
\mathrm{G} 2=24 \pm 4 \text { anos })\end{array}$ & $M=14$ & $60 \mathrm{~min}$ & $\mathrm{G} 1=5 ; \mathrm{G} 2=6$ & 3 & 6 e 12 & $\begin{array}{l}\text { Máxima (6RM) e } \\
50 \% 6 \mathrm{RM}(12)\end{array}$ & $\begin{array}{c}\mathrm{G} 1=\text { redução na PAS durante } 50 \text { min após } \\
\text { 6RM e durante } 40 \text { min após circuito } \\
\text { G2 = reduçáo na PAS durante } 60 \text { min após } \\
\text { 6RM e durante } 40 \text { min após } 12 \text { rep; redução } \\
\text { na PAD durante } 10 \text { min após } 12 \text { rep } \\
\end{array}$ \\
\hline MacDonald et al. ${ }^{26}$ & Ativos (24 \pm 2 anos) & $M=13$ & $60 \mathrm{~min}$ & 1 & $\begin{array}{l}15 \text { min de } \\
\text { execução }\end{array}$ & Máximas & $65 \%$ 1RM & Redução na PAS entre 10 e 60 min \\
\hline Fisher $^{6}$ & Ativos (45 \pm 2 anos) & $F=9$ & $60 \mathrm{~min}$ & 5 (circuito) & 3 & 15 & $50 \% 1 \mathrm{RM}$ & Redução na PAS nos 60 min \\
\hline \multirow[t]{2}{*}{ Brown et al..27 } & Ativos (21 \pm 2 anos) & $M=5 ; F=2$ & $60 \mathrm{~min}$ & 5 & 3 & $8-10$ e $20-25$ & $\begin{array}{l}70 \% 1 \mathrm{RM}(8-10) \text { e } \\
40 \% 1 \mathrm{RM}(20-25)\end{array}$ & NS \\
\hline & Hipertensos & & & & & & & \\
\hline Hardy e Tucker ${ }^{28}$ & $\begin{array}{l}\text { Sedentários } \\
(51 \pm 10 \text { anos })\end{array}$ & $M=24$ & $24 \mathrm{~h}$ & 7 & 3 & $8-12$ & Máxima (8-12 RM) & Redução na PAS durante 1h pós-exercício \\
\hline Melo et al. ${ }^{4}$ & Sedentários (46 \pm 1 anos) & $F=11$ & $21 \mathrm{~h}$ & 6 & 3 & 20 & $40 \% 1 \mathrm{RM}$ & $\begin{array}{l}\text { Redução na PAS, PAD e PAM durante o } \\
\text { período de vigília }\end{array}$ \\
\hline Melo et al. ${ }^{4}$ & Sedentários (46 \pm 1 anos) & $F=11$ & $120 \mathrm{~min}$ & 6 & 3 & 20 & $40 \% 1 \mathrm{RM}$ & $\begin{array}{l}\text { Redução na PAS durante } 90 \text { min; redução } \\
\text { na PAD entre } 45 \text { e } 75 \text { min pós-exercício }\end{array}$ \\
\hline Fisher $^{6}$ & Ativos ( $48 \pm 3$ anos) & $F=7$ & $60 \mathrm{~min}$ & 5 (circuito) & 3 & 15 & $50 \% 1 \mathrm{RM}$ & Redução na PAS nos 60 min \\
\hline
\end{tabular}

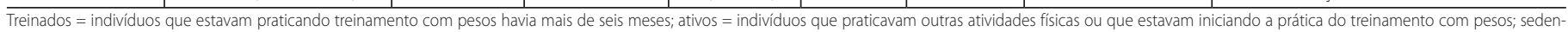

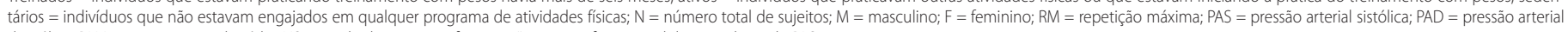
diastólica; PAM = pressão arterial média; NS = resultados não significativos; ${ }^{*}$ somente foram medidos os valores de PAS.

PAS e PAD, mas sem interação com o treinamento ${ }^{(33)}$. A tabela 4 ilustra o comportamento da PA enquanto a tabela 5 mostra o delineamento do treinamento utilizado nos estudos.

Considerando a prescrição do treinamento com pesos, observou-se que a maioria dos experimentos utilizou delineamento ajustado para as recomendações atuais ${ }^{(9,13)}$. Somente um estudo adotou como forma de treinamento o exercício estático ${ }^{(31)}$. Nesse experimento, mesmo reduzindo PAS e PAD de repouso, não houve alusão ao comportamento da força muscular pós-treino, diminuindo o poder de discussão sobre essa estratégia de prescrição como forma de aumentar a força muscular destinada à qualidade de vida. Considerando as referências sobre treinamento com pesos e comportamento da PA de repouso em normotensos, é esperado significativo aumento da força muscular. Tal aumento correlaciona-se com a intensidade do treinamento. Por exemplo, o estudo de Van Hoof et al. ${ }^{(34)}$, por a carga de esforço ser relativamente elevada, ocasionou maior aumento da força muscular que intensidades mais baixas ${ }^{(35)}$. Mas, independentemente do comportamento da força muscular com o treino, reduções de repouso na PAS e na PAD ocorreram na menor parte das pesquisas, conotando uma relação inversa entre a qualidade física e a variável fisiológica. Ou seja, a força deve aumentar, mas a PA não necessariamente vai diminuir.

Em relação aos estudos com hipertensos, quatro reportaram aumento significativo na força (5,32,40,41); três, redução na PAS(5,41,43); dois, redução na $P A D^{(5,40)}$. Somente um estudo mostrou redução na PAS e PAD e aumento na força ${ }^{(5)}$. Contudo, esse referido experimento teve como objetivo prin- cipal analisar o comportamento glicêmico de idosos diabéticos. Para tal, a amostra (grupo experimental e controle) foi submetida a controle alimentar, o que pode ter influenciado os resultados. Quanto aos demais estudos, uma referência mostrou redução na PAS e PAD, mas também houve redução semelhante no grupo controle(42). Assim, não se pôde inferir sobre o efeito do treinamento nessa amostra. Em relação à prescrição do treinamento com pesos, a maioria dos estudos envolvendo hipertensos que identificaram redução na PA de repouso seguiu as recomendações atuais ${ }^{(9,13)}$. Somente um experimento utilizou exercício estático ${ }^{(43)}$. Embora os autores reportassem redução significativa na PAS de repouso, não houve alusão ao comportamento da força muscular. Além disso, não é recomendável para hipertensos o treinamento com alto componente estático(1), o que diminui a validade externa no estudo. De forma semelhante ao que foi relatado em amostras normotensas, o treinamento com pesos aplicado em hipertensos apresenta grande possibilidade de aumentar a força muscular. No entanto, reduções em PAS e PAD de repouso ainda são duvidosas, carecendo de maiores investigações.

\section{Possíveis mecanismos}

A PA é mediada pela interação entre débito cardíaco e resistência vascular periférica. Assim, a redução da PA está intimamente relacionada com a diminuição simultânea ou isolada dessas duas variáveis. Contudo, poucos foram os estudos que se propuseram a investigar os mecanismos relacionados com a redução da PA de repouso após uma sessão de exercícios com pesos ou um treinamento da mesma natureza. 
Tabela 4. Comportamento da pressão arterial sistólica (PAS) e diastólica (PAD) de repouso após o treinamento com pesos

\begin{tabular}{|c|c|c|c|c|c|c|c|c|}
\hline \multirow{2}{*}{ Referência } & \multirow{2}{*}{ Amostra } & \multirow{2}{*}{ Idade (anos) } & \multirow{2}{*}{$\begin{array}{l}\text { Estado de } \\
\text { treinamento }\end{array}$} & \multirow{2}{*}{ Duração } & \multicolumn{2}{|c|}{ PAS (mmHg) } & \multicolumn{2}{|c|}{ PAD (mmHg) } \\
\hline & & & & & Antes & Depois & Antes & Depois \\
\hline \multicolumn{9}{|l|}{ NORMOTENSOS } \\
\hline \multicolumn{9}{|l|}{ Olson et al. ${ }^{29}$} \\
\hline Experimental & $F=15$ & $39,0 \pm 1,0$ & Sedentários & 1 ano & $119,0 \pm 3,0$ & $104,0 \pm 7,0$ & $69,0 \pm 3,0$ & $62,0 \pm 5,0$ \\
\hline Controle & $F=15$ & $39,0 \pm 2,0$ & Sedentários & & $119,0 \pm 2,0$ & $117,0 \pm 3,0$ & $67,0 \pm 2,0$ & $68,0 \pm 2,0$ \\
\hline \multicolumn{9}{|l|}{ Anton et al..$^{30}$} \\
\hline Experimental & $M=3 ; F=10$ & $52,0 \pm 2,0$ & Sedentários & 13 semanas & $112,0 \pm 3,0$ & $112,0 \pm 3,0$ & $65,0 \pm 2,0$ & $63,0 \pm 2,0$ \\
\hline Controle & $M=4 ; F=9$ & $53,0 \pm 2,0$ & Sedentários & & $120,0 \pm 4,0$ & $118,0 \pm 4,0$ & $65,0 \pm 3,0$ & $66,0 \pm 3,0$ \\
\hline \multicolumn{9}{|l|}{ Carter et al. ${ }^{7}$} \\
\hline Experimental & $M=11 ; F=1$ & $21,0 \pm 0,3$ & Ativos & 8 semanas & $130,0 \pm 3,0$ & $121,0 \pm 2,0$ & $69,0 \pm 3,0$ & $61,0 \pm 2,0$ \\
\hline Controle & $M=11 ; F=2$ & $21,0 \pm 0,8$ & Ativos & & $119,0 \pm 3,0$ & $120,0 \pm 3,0$ & $64,0 \pm 2,0$ & $62,0 \pm 2,0$ \\
\hline \multicolumn{9}{|l|}{ Wiley et al. ${ }^{31}$} \\
\hline Experimental & 8 & $20-35$ & Ativos & 8 semanas & $134,1 \pm 0,1$ & $121,4 \pm 1,3$ & $86,5 \pm 2,0$ & $71,6 \pm 3,5$ \\
\hline Controle & 7 & $20-35$ & Ativos & & $134,0 \pm 3,3$ & $136,6 \pm 2,8$ & $83,4 \pm 1,7$ & $85,0 \pm 2,4$ \\
\hline \multicolumn{9}{|l|}{ Cononie et al. ${ }^{32}$} \\
\hline Experimental & $\mathrm{MeF}=14$ & $70-79$ & Sedentários & 26 semanas & $122,0 \pm 8,0$ & $122,0 \pm 11,0$ & $76,0 \pm 9,0$ & $75,0 \pm 10,0$ \\
\hline Controle & $\mathrm{MeF}=7$ & 70-79 & Sedentários & & $126,0 \pm 7,0$ & $129,0 \pm 7,0$ & $78,0 \pm 6,0$ & $81,0 \pm 5,0$ \\
\hline \multicolumn{9}{|l|}{ Katz e Wilson ${ }^{33}$} \\
\hline Experimental & $F=13$ & $18-27$ & Sedentários & 6 semanas & $107,5 \pm 11,6$ & $99,1 \pm 13,6$ & $65,3 \pm 6,8$ & $61,2 \pm 7,8$ \\
\hline Controle & $F=13$ & $18-22$ & Sedentários & & $113,8 \pm 8,3$ & $112,5 \pm 5,8$ & $67,2 \pm 6,0$ & $68,2 \pm 6,7$ \\
\hline \multicolumn{9}{|l|}{ Vanhoof et al..$^{34}$} \\
\hline Experimental & $M=8$ & $?$ & Sedentários & 48 sessões & $129,0 \pm 8,0$ & $125,0 \pm 6,0$ & $81,0 \pm 10,0$ & $76,0 \pm 5,0$ \\
\hline Controle & $M=11$ & $?$ & Sedentários & & $124,0 \pm 15,0$ & $120,0 \pm 9,0$ & $78,0 \pm 14,0$ & $76,0 \pm 11,0$ \\
\hline Tsutsumi et al..$^{35}$ & & & & & & & & \\
\hline Experimental (a) & $M=11 ; F=2$ & $67,8 \pm 4,9$ & Sedentários & 12 semanas & $109,8 \pm 18,8$ & $103,7 \pm 17,4$ & $65,0 \pm 9,9$ & $62,3 \pm 9,9$ \\
\hline Experimental (b) & $M=11 ; F=3$ & $68,9 \pm 7,5$ & Sedentários & & $124,2 \pm 16,4$ & $110,8 \pm 15,0$ & $72,6 \pm 9,5$ & $67,5 \pm 9,1$ \\
\hline Controle & $M=11 ; F=3$ & $69,8 \pm 4,6$ & Sedentários & & $122,0 \pm 11,8$ & $125,4 \pm 14,1$ & $72,4 \pm 8,1$ & $76,0 \pm 9,8$ \\
\hline Wood et al. ${ }^{36}$ & & & & & & & & \\
\hline Experimental & $M=5 ; F=5$ & $69,8 \pm 6,0$ & Pouco ativos & 12 semanas & $129,1 \pm 22,5$ & $124,1 \pm 16,3$ & $75,1 \pm 10,3$ & $72,6 \pm 10,6$ \\
\hline Controle & $M=3 ; F=3$ & $68,0 \pm 5,4$ & Pouco ativos & & $133,5 \pm 22,4$ & $129,7 \pm 16,5$ & $78,3 \pm 6,9$ & $80,3 \pm 8,8$ \\
\hline Vincent et al. ${ }^{37}$ & & & & & & & & \\
\hline Experimental (a) & $M e F=22$ & $67,6 \pm 6,0$ & Pouco ativos & 24 semanas & $137,8 \pm 17,0$ & $138,9 \pm 15,0$ & $80,7 \pm 9,0$ & $83,4 \pm 6,0$ \\
\hline Experimental (b) & $\mathrm{Me} F=24$ & $66,6 \pm 7,0$ & Pouco ativos & 24 semanas & $132,9 \pm 10,0$ & $129,7 \pm 9,0$ & $83,8 \pm 8,0$ & $81,1 \pm 10,1$ \\
\hline Controle & $M e F=16$ & $71,0 \pm 5,0$ & Pouco ativos & & $130,2 \pm 16,0$ & $129,3 \pm 19,0$ & $78,2 \pm 10,0$ & $79,5 \pm 12,0$ \\
\hline Sigal et al. ${ }^{38}$ & & & & & & & & \\
\hline Experimental & $M=40 ; F=24$ & $54,7 \pm 7,5$ & Sedentários & 22 semanas & $136,0 \pm 22,0$ & $131,0 \pm 23,0$ & $80,0 \pm 13,0$ & $78,0 \pm 14,0$ \\
\hline Controle & $M=41 ; F=22$ & $54,8 \pm 7,2$ & Sedentários & & $133,0 \pm 20,0$ & $129,0 \pm 21,0$ & $80,0 \pm 12,0$ & $79,0 \pm 13,0$ \\
\hline Myiachi et al. ${ }^{39}$ & & & & & & & & \\
\hline Experimental & $M=14$ & $22,0 \pm 1,0$ & Sedentários & 4 meses & $116,0 \pm 3,0$ & $116,0 \pm 3,0$ & $69,0 \pm 1,0$ & $66,0 \pm 1,0$ \\
\hline Controle & $M=14$ & $22,0 \pm 1,0$ & Sedentários & & $118,0 \pm 3,0$ & $120,0 \pm 2,0$ & $69,0 \pm 2,0$ & $72,0 \pm 2,0$ \\
\hline HIPERTENSOS & & & & & & & & \\
\hline Harrys e Holly ${ }^{40}$ & & & & & & & & \\
\hline Experimental & $M=10$ & $32,7 \pm 5,2$ & Sedentários & 9 semanas & $141,7 \pm 7,9$ & $142,3 \pm 7,5$ & $95,8 \pm 6,4$ & $91,3 \pm 3,8$ \\
\hline Controle & $M=16$ & $31,4 \pm 6,2$ & Sedentários & & $141,6 \pm 8,2$ & $145,8 \pm 6,9$ & $94,6 \pm 3,8$ & $92,6 \pm 3,3$ \\
\hline Dunstan et al. ${ }^{5}$ & & & & & & & & \\
\hline Experimental & $M=10 ; F=6$ & $67,6 \pm 5,2$ & Sedentários & 6 meses & $145,0 \pm 17,8$ & $-6,7 \pm 10,0$ & $78,0 \pm 8,8$ & $-4,4 \pm 6,9$ \\
\hline Controle & $M=6 ; F=7$ & $66,9 \pm 5,3$ & Sedentários & & $147,0 \pm 15,5$ & $-2,5 \pm 15,8$ & $75,0 \pm 6,4$ & $-0,9 \pm 10,1$ \\
\hline Castaneda et al. ${ }^{41}$ & & & & & & & & \\
\hline Experimental & $M=10 ; F=21$ & $66,0 \pm 2,0$ & Sedentários & 16 semanas & $145,2 \pm 3,6$ & $135,5 \pm 3,3$ & $72,6 \pm 1,1$ & $71,1 \pm 2,1$ \\
\hline Controle & $M=12 ; F=19$ & $66,0 \pm 1,0$ & Sedentários & & $142,7 \pm 4,1$ & $150,4 \pm 3,9$ & $69,2 \pm 1,2$ & $70,8 \pm 1,4$ \\
\hline Cononie et al..$^{32}$ & & & & & & & & \\
\hline Experimental & $M e F=6$ & $70-79$ & Sedentários & 26 semanas & $151,0 \pm 7,0$ & $151,0 \pm 11,0$ & $82,0 \pm 9,0$ & $82,0 \pm 14,0$ \\
\hline Controle & $M$ e $F=5$ & 70-79 & Sedentários & & $153,0 \pm 7,0$ & $156,0 \pm 10,0$ & $85,0 \pm 8,0$ & $85,0 \pm 6,0$ \\
\hline Blumenthal et al. ${ }^{42}$ & & & & & & & & \\
\hline Experimental & $M=18$ e $F=13$ & $46,0 \pm 7,0$ & Sdentários & 16 semanas & $143,0 \pm 10,3$ & $136,0 \pm 11,6$ & $95,0 \pm 5,4$ & $89,0 \pm 6,4$ \\
\hline Controle & $M=15$ e $F=7$ & $45,7 \pm 7,8$ & Sedentários & & $142,0 \pm 12,0$ & $133,0 \pm 8,6$ & $95,0 \pm 6,2$ & $90,0 \pm 6,2$ \\
\hline Taylor et al. ${ }^{43}$ & & & & & & & & \\
\hline Experimental & $M=5 ; F=4$ & $69,3 \pm 6,0$ & Ativos & 10 semanas & $156,0 \pm 9,4$ & $137,0 \pm 7,8$ & $82,3 \pm 9,3$ & $75,0 \pm 10,9$ \\
\hline Controle & $M=5 ; F=3$ & $64,2 \pm 5,5$ & Ativos & & $152,0 \pm 7,8$ & $144,0 \pm 11,8$ & $87,1 \pm 10,8$ & $84,0 \pm 9,6$ \\
\hline
\end{tabular}

$\mathrm{M}=$ masculino; $\mathrm{F}=$ feminino; $\mathrm{MS}=$ membros superiores; $\mathrm{MI}=$ membros inferiores; ${ }^{*}$ quantidade de carga em todos os exercícios; Sedentários = sujeitos não praticantes de qualquer atividade fisica regular; Pouco ativos = praticantes de atividades físicas em frequência inferior a duas vezes semanais; ativos = praticantes de atividades físicas em frequência superior a duas vezes semanais 
Tabela 5. Delineamento do treinamento com pesos em relação aos estudos que investigaram o comportamento da pressão arterial de repouso

\begin{tabular}{|c|c|c|c|c|c|c|c|}
\hline \multirow{2}{*}{ Referência } & \multirow{2}{*}{$\begin{array}{l}\text { Número de } \\
\text { exercícios }\end{array}$} & \multirow{2}{*}{ Séries } & \multirow{2}{*}{ Repetições } & \multirow{2}{*}{ Intensidade (\% 1RM) } & \multirow{2}{*}{$\begin{array}{c}\text { Frequência } \\
\text { semanal }\end{array}$} & \multicolumn{2}{|c|}{ Força } \\
\hline & & & & & & Antes & Depois \\
\hline \multicolumn{8}{|l|}{ NORMOTENSOS } \\
\hline \multicolumn{8}{|l|}{ Olson et al } \\
\hline Experimental & Não informado & 3 & $8-12$ & Não informado & $\begin{array}{c}\text { Mínimo de } \\
\text { duas }\end{array}$ & $\begin{aligned} 1 \mathrm{RM} \text { no supino } & =37,9 \pm 1,5 \mathrm{~kg} ; \\
1 \mathrm{RM} \text { no leg-press } & =123,9 \pm 4,9 \mathrm{~kg}\end{aligned}$ & $\begin{aligned} 1 \mathrm{RM} \text { no supino } & =41,2 \pm 1,7 \mathrm{~kg} ; \\
1 \mathrm{RM} \text { no leg-press } & =142,7 \pm 7,4 \mathrm{~kg}\end{aligned}$ \\
\hline Controle & & & & & & $\begin{aligned} 1 \text { RM no supino } & =37,9 \pm 2,2 \mathrm{~kg} ; \\
1 \mathrm{RM} \text { no leg-press } & =121,3, \pm 7,7 \mathrm{~kg}\end{aligned}$ & $\begin{aligned} 1 \mathrm{RM} \text { no supino } & =37,1 \pm 1,9 \mathrm{~kg} ; \\
1 \mathrm{RM} \text { no leg-press } & =137,6 \pm 8,4 \mathrm{~kg}\end{aligned}$ \\
\hline \multicolumn{8}{|l|}{ Anton et al. } \\
\hline Experimental & 8 & 2 & Máximas & $\begin{array}{l}1^{\text {a }} \text { série }=50 \% 1 \mathrm{RM} ; \\
2^{\mathrm{a}} \text { série }=75 \% 1 \mathrm{RM}\end{array}$ & 3 & Não informado & Aumento em 1 RM entre 26-35\% \\
\hline Controle & & & & & & Não testado & Não testado \\
\hline \multicolumn{8}{|l|}{ Carter et al. } \\
\hline Experimental & 7 & 3 & $\begin{array}{l}1^{\mathrm{a}} \text { e } 2^{\mathrm{a}} \text { séries }=10 ; \\
3^{\mathrm{a}} \text { série = máxima }\end{array}$ & Não informado & 3 & & Aumento em 1 RM entre $16,7-42,5 \%$ \\
\hline Controle & & & & & & & 1 RM entre - 1,6 e + 2,4\% \\
\hline \multicolumn{8}{|l|}{ Wiley et al. } \\
\hline Experimental & 1 (estático) & 4 & 2 min de contração & 30 & 3 & Não informado & Não informado \\
\hline Controle & & & & & & Não informado & Não informado \\
\hline \multicolumn{8}{|l|}{ Cononie et al. ${ }^{+}$} \\
\hline Experimental & 10 & 1 & $8-12$ & Progressiva até 8-12 RM & 3 & $\mathrm{MS}=38,5 \pm 15,0 \mathrm{~kg}^{*} ; \mathrm{Ml}=48,9 \pm 18,6 \mathrm{~kg}^{*}$ & $M S=45,4 \pm 18,1 \mathrm{~kg} ; \mathrm{Ml}=53,4 \pm 21,1 \mathrm{~kg}$ \\
\hline Controle & & & & & & $\mathrm{MS}=34,3 \pm 18,2 \mathrm{~kg}^{*} ; \mathrm{Ml}=45,7 \pm 19,5 \mathrm{~kg}^{*}$ & $M S=32,0 \pm 21,1 \mathrm{~kg} ; \mathrm{Ml}=43,2 \pm 20,3 \mathrm{~kg}$ \\
\hline \multicolumn{8}{|l|}{ Katz e Wilson } \\
\hline Experimental & 14 (circuito) & 1 & $\mathrm{MS}=11-12 ; \mathrm{Ml}=14-15$ & 30 & 3 & $\mathrm{MS}=71,2 \mathrm{~kg}^{*} ; \mathrm{Mi}=118,8 \mathrm{~kg}^{*}$ & $\mathrm{MS}=+31 \% ; \mathrm{Ml}=+26 \%$ \\
\hline Controle & & & & & & Não informado & Não informado \\
\hline \multicolumn{8}{|l|}{ Vanhoof et al. } \\
\hline Experimental & 6 & 3 & $10-12$ & $70-90$ & 3 & & Aumento em 1 RM entre $24-95 \%$ \\
\hline Controle & & & & & & Não informado & Não informado \\
\hline \multicolumn{8}{|l|}{ Tsutsumi et al. } \\
\hline Experimental (a) & 12 & 2 & $8-12$ & $75-85$ & 3 & $10 \mathrm{RM}$ em MS $=19,7 \pm 5,5 \mathrm{~kg}$ & $+48,2 \%$ \\
\hline Experimental (b) & 12 & 2 & $12-16$ & $55-65$ & 3 & $10 \mathrm{RM}$ em MS $=19,8 \pm 6,9 \mathrm{~kg}$ & $+39,7 \%$ \\
\hline Controle & & & & & & $10 \mathrm{RM}$ em MS $=22,3 \pm 8,2 \mathrm{~kg}$ & $-1,0 \%$ \\
\hline \multicolumn{8}{|l|}{ Wood et al. } \\
\hline Experimental & 8 & 2 & $8-12$ & Máxima para as repetições & 3 & Não informado & Aumento em 5 RM entre $36,6-63,5 \%$ \\
\hline Controle & & & & & & Não informado & 5 RM entre $-2,5 \%$ e $+4,4 \%$ \\
\hline Vincent et al. & & & & & & & \\
\hline Experimental (a) & 13 & 1 & 13 & 50 & 3 & Não informado & Aumento em 1 RM entre 10,8-25,3\% \\
\hline Experimental (b) & 13 & 1 & 8 & 80 & 3 & Não informado & Aumento em 1 RM entre $14,6-27,6 \%$ \\
\hline Controle & & & & & & Não informado & Não informado \\
\hline Sigal et al. & & & & & & & \\
\hline Experimental & 7 & $2-3$ & $7-9$ & Máxima para as repetições & 3 & Não informado & Não informado \\
\hline Controle & & & & & & Não informado & Não informado \\
\hline Myiachi et al. & & & & & & & \\
\hline Experimental & 8-12 & 3 & 12 & 80 & 3 & Não informado & Aumento em 1 RM entre 20-47\% \\
\hline Controle & & & & & & Não testado & Não testado \\
\hline HIPERTENSOS & & & & & & & \\
\hline Harrys e Holly & & & & & & & \\
\hline Experimental & 10 (circuito) & 3 & $20-25$ & 40 & 3 & $\begin{aligned} 1 \mathrm{RM} \text { no supino } & =57 \pm 3 \mathrm{~kg} \\
1 \mathrm{RM} \text { no leg-press } & =134 \pm 8 \mathrm{~kg}\end{aligned}$ & $\begin{array}{c}\text { Aumento de } 12,3 \% \text { no supino } \\
\text { e } 53,0 \% \text { no leg-press }\end{array}$ \\
\hline Controle & & & & & & $?$ & $?$ \\
\hline Dunstan et al. & & & & & & & \\
\hline Experimental & 9 & 3 & $8-10$ & $75-85$ & 3 & $?$ & $\begin{array}{c}\text { Aumento de } 43,2 \pm 34,2 \% \text { no MS } \\
\text { e de } 33 \pm 21,7 \% \text { no Ml }\end{array}$ \\
\hline Controle & & & & & & $?$ & $\begin{array}{c}\text { Aumento de } 1,5 \pm 17,7 \% \text { no MS } \\
\text { e de } 5,0 \pm 16,9 \% \text { no } \mathrm{Ml}\end{array}$ \\
\hline Castaneda et al. & & & & & & & \\
\hline Experimental & 5 & 3 & 8 & $60-80$ & 3 & $389 \pm 30 \mathrm{~kg}^{*}$ & $+33 \pm 7 \%$ \\
\hline Controle & & & & & & $351 \pm 31 \mathrm{~kg}^{*}$ & $-15 \pm 3 \%$ \\
\hline Blumenthal et al. & & & & & & & \\
\hline Experimental & ? (circuito) & $?$ & $?$ & $?$ & 3 & Não informado & Não informado \\
\hline Controle & & & & & & Não informado & Não informado \\
\hline Taylor et al. & & & & & & & \\
\hline Experimental & 1 (estático) & $2 \mathrm{~min}$ & - & 30 & 3 & Não informado & Não informado \\
\hline Controle & & & & & & Não informado & Não informado \\
\hline
\end{tabular}


No caso da HPE decorrente da atividade com pesos, somente dois experimentos buscaram possíveis explicações para o comportamento da PA pós-esforço. O estudo de MacDonald et al.(26), por exemplo, não encontrou associação entre os valores de peptídio atrial natriurético com a ocorrência da HPE após uma sessão de exercício com pesos. Contudo, essa substância, embora associada ao comportamento hemodinâmico, não deve apresentar isoladamente efeito relevante na redução da PA após exercício(44). Mais recentemente, Rezk et al. ${ }^{(24)}$, observaram que a redução na PA após o exercício com pesos deve-se, pelo menos em parte, à diminuição no débito cardíaco. Esse comportamento no débito cardíaco relacionou-se com a queda no volume sistólico e aumento compensatório na frequência cardíaca. Tais dados sugerem ação simpática no coração. Porém, faltam experimentos a fim de relacionar possíveis agentes vasodilatadores dependentes do endotélio, tais como óxido nítrico e prostaglandinas, nesse comportamento.

Quanto ao treinamento sistemático do exercício com pesos, poderse-ia especular alguma modificação na atividade nervosa simpática com a resposta da PA de repouso. Em se tratando de pessoas jovens e normotensas, o estudo de Carter et al. ${ }^{(7)}$ identificou redução significativa na PAS e PAD, mas sem qualquer alteração na atividade nervosa simpática. Por outro lado, Taylor et al. ${ }^{(43)}$, com amostra de idosos hipertensos, identificaram redução na PAS de repouso e melhora na modulação simpatovagal. Esses resultados pressupõem que pessoas normotensas não sofreriam modificações no comportamento simpático, ao contrário dos indivíduos hipertensos. Dessa forma, outros mecanismos estariam relacionados com a redução da PA. Por exemplo, a condutância vascular reduziu-se após 13 semanas de treinamento com pesos em normotensos, embora a PA não tenha se modificado. Não obstante, os diferentes delineamentos experimentais, características da amostra e duração do treinamento não permitem, por enquanto, inferir com maior precisão os mecanismos relacionados com a redução da PA decorrente do treinamento com pesos.

\section{CONCLUSÃO}

Atualmente, recomenda-se o treinamento com pesos associado ao treinamento aeróbio para a saúde e a qualidade de vida, seja de pessoas portadoras $^{(1,2)}$ ou não ${ }^{(16)}$ de doenças crônicas. Contudo, são prescrições isoladas que merecem maior atenção, principalmente quando a amostra é composta por indivíduos com fatores de risco de doença cardiovascular, uma vez que a PA pode se elevar significativamente durante essa prática e em curto período de tempo ${ }^{(45)}$. Porém, o aumento da força muscular é importante para a qualidade de vida, pois pode representar menor esforço cardiovascular quando há a necessidade de se mobilizar certa carga ${ }^{(8)}$. Dessa forma, seria adequado que o treinamento com pesos aumentasse a força muscular em amostras hipertensas, por exemplo. Mais ainda, se esse modelo de treino reduzisse valores de PA de repouso, constituiria mais uma forma de reduzir os riscos de PA elevada. Porém, com base na presente revisão, não se pode concluir que o treinamento com pesos seja realmente eficiente para reduzir a PA de repouso, tanto de forma crônica ou momentânea. Embora alguns estudos mostrem resultados promissores, novas investigações são necessárias para estabelecer o real papel do exercício físico de força sobre a PA.

Todos os autores declararam não haver qualquer potencial conflito de interesses referente a este artigo.

\section{REFERÊNCIAS BIBLIOGRÁFICAS}

1. American College of Sports Medicine Position Stand. Exercise and hypertension. Med Sci Sports Exerc 2004;36:533-53.

2. V Diretrizes Brasileiras de Hipertensão. Arq Bras Cardiol 2007;89:e24-79.

3. Kenney MJ, Seals DR. Post exercise hypotension. Key features, mechanisms, and clinical significance. Hypertension 1993:22:653-64.

4. Melo CM, Alencar Filho AC, Tinucci T, Mion Jr D, Forjaz CL. Postexercise hypotension induced by low-intensity resistance exercise in hypertensive women receiving captopril. Blood Press Monit 2006;11:183-9.

5. Dunstan DW, Daly RM, Owen N, Jolley D, De Courten M, Shaw J, et al. High-intensity resistance training improves glycemic control in older patients with type 2 diabetes. Diabetes Care 2002;25:1729-36.

6. Fisher MM. The effect of resistance exercise on recovery blood pressure in normotensive and borderline hipertensive women. J Strength Cond Res 2001;15:210-6.

7. Carter JR, Ray CA, Downs EM, Cooke WH. Strength training reduces arterial blood pressure but not sympathetic neural activity in young normotensive subjects. J Appl Physiol 2003;94:2212-6.

8. McCartney N, McKelvie RS, Martin J, Sale DG, MacDougall JD. Weight-training-induced attenuation of the circulatory response of older males to weight lifting. J Appl Physiol 1993;74:1056-60.

9. American College of Sports Medicine Position Stand. Progression models in resistance training for healthy adults. Med Sci Sports Exerc 2002;34:364-80.

10. Simão R, Fleck S, Polito MD, Monteiro WD, Farinatti PTV. Effects of resistance training intensity, volume, and session format on the postexercise hypotensive response. J Strength Cond Res 2005;19:853-8.

11. Kelley GA, Kelley KS. Progressive resistance exercise and resting blood pressure: a meta-analysis of randomized controlled trials. Hypertension 2000;35:838-43.

12. Cornelissen VA, Fagard RH. Effect of resistance training on resting blood pressure: a meta-analysis of randomized controlled trials. J Hypertens 2005;23:251-9.

13. Rhea MR, Alvar BA, Burkett LN, Ball SD. A meta-analysis to determine the dose response for strength development. Med Sci Sports Exerc 2003;35:456-64.

14. Kraemer WJ, Ratamess NA. Fundamentals of resistance training: progression and exercise prescription. Med Sci Sports Exerc 2004;36:674-88

15. Suzuki T, Bean JF, Fielding RA. Muscle power of the ankle flexors predicts functional performance in community-dwelling older women. J Am Geriatr Soc 2001;49:1161-67.

16. ACSM Position Stand on The recommended quantity and quality of exercise for developing and maintaining cardiorespiratory and muscular fitness, and flexibility in adults. Med Sci Sports Exerc 1998;30:975-91.

17. Gabriel DA, Kamen G, Frost G. Neural adaptations to resistive exercise. Mechanisms and recommendations for training practices. Sports Med 2006;36:133-49.

18. Roltsch MH, Mendez T, Wilund KR, Hagberg JM. Acute resistive exercise does not affect ambulatory blood pressure in young men and women. Med Sci Sports Exerc 2001;33:881-6.

19. Bermudes AMLM, Vassallo DV, Vasquez EC, Lima EG. Monitorização ambulatorial da pressão arterial em indivíduos normotensos submetidos a duas sessões únicas de exercícios: resistido e aeróbio. Arq Bras Cardiol 2004;82:57-64

20. Niemelä TH, Kiviniemi AM, Hautala AJ, Salmi JA, Linnamo V, Tulppo MP. Recovery pattern of baroreflex sensitivity after exercise. Med Sci Sports Exerc 2008;40:864-70.

21. Focht BC, Koltyn KF. Influence of resistance exercise of different intensities on state anxiety and blood pressure. Med Sci Sports Exerc 1999;31:456-63.

22. DeVan AE, Anton MM, Cook JN, Neidre DB, Cortez-Cooper MY, Tanaka H. Acute effects of resistance exercise on arterial compliance. J Appl Physiol 2005;98:2287-91.

23. O'Connor PJ, Bryant CX, Veltri JP, Gebhardt SM. State anxiety and ambulatory blood pressure following resistance exercise in females. Med Sci Sports Exerc 1993;25:516-21
24. Rezk CC, Marrache RC, TinucciT, Mion D Jr, Forjaz CL. Post-resistance exercise hypotension, hemodynamics, and heart rate variability: influence of exercise intensity. Eur J Appl Physiol 2006;98:105-12.

25. Hill DW, Collins MA, Cureton KJ, DeMello J. Blood pressure response after weight training exercise. J Appl Sports Sci Res 1989;3:44-7.

26. MacDonald JR, MacDougall JD, Interisano SA, Smith KM, McCartney N, Moroz JS, et al. Hypotension following mild bouts of resistance exercise and submaximal dynamic exercise. Eur J Appl Physiol Occup Physiol 1999;79:148-54.

27. Brown SP, Clemons JM, He Q, Liu S. Effects of resistance exercise and cycling on recovery blood pressure. J Sports Sci 1994;12:463-8.

28. Hardy DO, Tucker LA. The effects of a single bout of strength training on ambulatory blood pressure levels in 24 mildly hypertensive men. Am J Health Promot 1998;13:69-72.

29. Olson TP, Dengel DR, Leon AS, Schmitz KH. Moderate resistance training and vascular health in overweight women. Med Sci Sports Exerc 2006;38:1558-64.

30. Anton MM, Cortez-Cooper MY, DeVan AE, Neidre DB, Cook JN, Tanaka H. Resistance training increases basal limb blood flow and vascular conductance in aging humans. J Appl Physiol 2006;101:1351-5.

31. Wiley RL, Dunn CL, Cox RH, Hueppchen NA, Scott MS. Isometric exercise training lowers resting blood pressure. Med Sci Sports Exerc 1992;24:749-54.

32. Cononie CC, Graves JE, Pollock ML, Phillips MI, Sumners C, Hagberg JM. Effect of exercise training on blood pressure in 70-79-yr-old men and women. Med Sci Sports Exerc 1991;23:505-11.

33. Katz J, Wilson BRA. The effects of a six-week, low-intensity nautilus circuit training program on resting blood pressure in females. J Sports Med Phys Fitness 1992;32:299-302.

34. Van Hoof R, Macor F, Lijnen P, Staessen J, Thijs L, Vanhees L, et al. Effect of strength training on blood pressure measured in various conditions in sedentary men. Int J Sports Med 1996;17:415-22.

35. Tsutsumi T, Don BM, Zaichkowsky LD, Delizonna LL. Physical fitness and psychological benefits of strength training in community dwelling older adults. Appl Human Sci 1997;16:257-66.

36. Wood RH, Reyes R, Welsch MA, Favaloro-Sabatier J, Sabatier M, Lee CM, et al. Concurrent cardiovascular and resistance training in healthy older adults. Med Sci Sports Exerc 2001;33:1751-58.

37. Vincent KR, Vincent HK, Braith RW, Bhatnagar V, Lowenthal DT. Strength training and hemodynamic responses to exercise. Am J Geriatr Cardiol 2003;12:97-106.

38. Sigal RJ, Kenny GP, Boulé NG, Wells GA, Prud'homme D, Fortier M, et al. Effects of aerobic training, resistance training, or both on glycemic control in type 2 diabetes. A randomized trial. Ann Intern Med 2007;147:357-69.

39. Miyachi M, Kawano H, Sugawara J, Takahashi K, Hayashi K, Yamazaki K, et al. Unfavorable effects of resistance training on central arterial compliance. A randomized intervention study. Circulation 2004;110:2858-63.

40. Harris KA, Holly RG. Physiological response to circuit weight training in borderline hypertensive subjects. Med Sci Sports Exerc 1987;19:246-52.

41. Castaneda C, Layne JE, Munoz-Orians L, Gordon PL, Walsmith J, Foldvari M, et al. A randomized controlled trial of resistance exercise training to improve glycemic control in older adults with type 2 diabetes. Diabetes Care 2002;25:2335-41.

42. Blumenthal JA, Siegel WC, Appelbaum M. Failure of exercise to reduce blood pressure in patients with mild hypertension. JAMA 1991;266:2098-104.

43. Taylor AC, McCartney N, Kamath MV, Wiley RL. Isometric training lowers resting blood pressure and modulates autonomic control. Med Sci Sports Exerc 2003;35:251-6.

44. Poveda JJ, Riestra A, Salas E, Cagigas ML, López-Somoza C, Amado JA, et al. Contribution of nitric oxide to exercise-induced changes in healthy volunteers: effects of acute exercise and long-term physical training. Eur J Clin Invest 1997;27:967-71.

45. Polito MD, Farinatti PTV, Lira VA, Nobrega ACL. Blood pressure assessment during resistance exercise: comparison between auscultation and Finapres. Blood Press Monit 2007;12:81-6. 